\title{
Transform Methods of Computerized Tomography in Studying Electronic Structure and Fermi Surfaces of Solids
}

\author{
G. Kontrym-SznAJD
}

Institute of Low Temperature and Structure Research

Polish Academy of Sciences, P.O. Box 1410, 50-950 Wrocław 2, Poland

We present mathematical methods of computerized tomography, based on an analytical inversion of the Radon transform either in terms of the Fourier transforms or series of orthogonal polynomials. These techniques, so-called transform methods, are discussed for reconstructing electronic densities from both line and plane projections measured either in the two-dimensional angular correlations of annihilation radiation or in onedimensional angular correlations of annihilation radiation and the Compton scattering experiments. This paper is devoted to review all of the papers where such techniques were applied for studying electronic momentum densities and the Fermi surface of solids by angular correlations of annihilation radiation and the Compton scattering experiments.

PACS numbers: 78.70.Bj, 71.18.+y, 87.59.Hp

\section{Introduction}

The problem of image reconstruction from both plane and line projections came into being independently in various scientific fields from radio astronomy, geology, physics and biology to medical diagnostics. First papers devoted to this question were published by Cormack in 1963 and 1964 [1] and by Mijnarends in 1967 [2]. Cormack found different mathematical solutions for line projections giving a proposal of their application in multiple X-ray tomography while Mijnarends found a solution for plane projections measured in one-dimensional (1D) angular correlations of annihilation radiation (ACAR) and Compton scattering for studying electronic structure of solids. Cormack's theory [1] and the first Xray tomograph made in 1972 by the British engineer Hounsfield revolutionized medical diagnostics — for which both scientists got the Nobel prize in 1979. In that time there was very fast development of various mathematical techniques of computerized tomography [3-6] as well as other techniques of medical diagnostics such as positron emission tomography (PET) and nuclear magnetic resonance (NMR). 
Yet earlier, the above mathematical questions were solved generally in 1917 by Radon [7], who considered a real function $\rho(\boldsymbol{p})$ in the $N$-dimensional space $R^{N}$ and its integrals over $(N-1)$-dimensional hyperplanes. Radon solved the following equation:

$$
\hat{R} \cdot \rho(\boldsymbol{p})=g(r, \boldsymbol{\zeta})=\int_{-\infty}^{\infty} \rho(\boldsymbol{p}) \delta(r-\boldsymbol{\zeta} \cdot \boldsymbol{p}) \mathrm{d} \boldsymbol{s}
$$

named the Radon transform of $\rho(\boldsymbol{p})$. $\boldsymbol{\zeta}$ is a unit vector in $R^{N}$ along $r$, where $r$ is the perpendicular distance of the hyperplane from the origin of the coordinate system. The equation $r=\boldsymbol{\zeta} \cdot \boldsymbol{p}$ defines the $(N-1)$-dimensional hyperplane. The original solution found by Radon (inverse transform being an integral of $(N-1)$ derivative of $g$ ) has many applications to partial differential equations [8].

In most experiments data are collected in such a way that the reconstruction of a 3D density from line projections can be reduced to a set of reconstructions of $2 \mathrm{D}$ densities, performed independently on succeeding parallel planes. This corresponds to the Radon transform with $N=2$, while $N=3$ corresponds to the reconstruction of $3 \mathrm{D}$ density from plane projections.

All reconstruction techniques can be classified into two different categories:

1. series expansion methods [9],

2. transform methods [10].

Series expansion reconstruction methods, known as algebraic techniques, iterative algorithms or optimization theory methods (e.g. quadratic or entropy optimization and Bayesian analysis) are based on the discretization of Eq. (1) at the beginning — instead of the line integral, a finite sum is considered. Transform methods consist in the analytical inversion of the Radon transform - here final results are "discretized" in the sense that the reconstructed function $\rho$ is determined for some finite set of points.

In this paper we present transform methods for the image reconstruction from $1 \mathrm{D}$ and 2D projections and corresponding solutions for $N=3$ giving a review of most papers where such techniques have been applied for studying electronic (or electron-positron) momentum densities and the Fermi surface of solids by 1D ACAR, 2D ACAR and Compton scattering experiments where from 1D spectra either fully $3 \mathrm{D}$ or $2 \mathrm{D}$ electron densities were reconstructed.

\section{Orthogonal polynomial methods}

Both functions $g$ and $\rho$ can be expanded into spherical harmonics $S_{l}$ defined on $R^{N}$ :

$$
\begin{aligned}
& g(r, \zeta)=\sum_{l} g_{l}(r) S_{l}(\zeta), \\
& \rho(p, \omega)=\sum_{l} \rho_{l}(p) S_{l}(\omega) .
\end{aligned}
$$

As was found by Deans [5], radial functions $g$ and $\rho$ are the Gegenbauer transform pair, i.e. 


$$
\begin{aligned}
& g_{1}(r)=c 1(l, \mu) \int_{r}^{\infty} p^{2 \mu} \rho_{1}(p) C_{1}^{\mu}(r / p)\left[1-(r / p)^{2}\right]^{\mu-1 / 2} \mathrm{~d} p, \\
& \rho_{1}(p)=c 2(l, \mu) \frac{1}{p} \int_{p}^{\infty} g_{1}^{(2 \mu+1)}(r) C_{1}^{\mu}(r / p)\left[(r / p)^{2}-1\right]^{\mu-1 / 2} \mathrm{~d} r,
\end{aligned}
$$

where $c 1=(4 \pi)^{\mu} c, c 2=(-1)^{2 \mu+1} c /\left(2 \pi^{\mu+1}\right)$ and $c=\Gamma(1+1) \Gamma(\mu) / \Gamma(1+2 \mu)$. Here $g^{(n)}$ denotes the $n$-th derivative of $g, C_{1}^{\mu}$ are the Gegenbauer polynomials and $\mu=N / 2-1$. For $N=3, \mu=1 / 2$ and $C_{l}^{\mu}=P_{l}$ while for $N=2, \mu=0$ and $C_{l}^{0}=\frac{2}{l} T_{l}$ where $P_{l}$ and $T_{l}$ denote the Legendre and 1st kind Chebyshev polynomials, respectively. Therefore, Eqs. (4) and (5) make general formulae (i.e. for $R^{N}$ ) of particular solutions found earlier by Cormack for $N=2$ and Mijnarends for $N=3$.

The singularity in Eq. (5) (as well as an estimation of derivative of the experimental quantity burdened with statistical noise) makes its application in numerical calculations difficult. This can be circumvented if $g$ is expanded into such orthogonal polynomials that $\rho$ is given as a series of other orthogonal polynomials, i.e. Eqs. (4), (5) are solved analytically. Here two different sets of orthogonal polynomials can be used.

\subsection{Gegenbauer and Jacobi polynomials}

The first set of polynomials is proposed by Luis [11]. If

$$
g_{l}(r)=\sum_{k=0}^{\infty} a_{l k}\left(1-r^{2}\right)^{\lambda-1 / 2} C_{l+2 k}^{\lambda}(r)
$$

(with $\lambda>N / 2-1$ ), then the radial parts of a density are given as follows:

$$
\rho_{l}(p)=\sum_{k=0}^{\infty} a_{l k} c(N, \lambda, k, l)\left(1-p^{2}\right)^{\lambda-N / 2} p^{l} P_{k}^{(\lambda-N / 2, l+n / 2-1)}\left(2 p^{2}-1\right),
$$

where $P_{k}^{\alpha, \beta}$ are the Jacobi polynomials and the coefficients $c(N, \lambda, k, l)$ read as

$$
c(N, \lambda, k, l)=2^{1-2 \lambda} \pi^{1-N / 2} \frac{\Gamma(l+2 k+2 \lambda) \Gamma(k+1)}{\Gamma(l+2 k+1) \Gamma(\lambda) \Gamma(k+1+\lambda-N / 2)} .
$$

For $N=2$ and $\lambda=1, C_{l+2 k}^{1}=U_{l+2 k}$ and $p^{l} P_{k}^{(0, l)}\left(2 p^{2}-1\right)=R_{l}^{k}(p)$ (Chebyshev 2nd kind and Zernike polynomials, respectively) which is the result derived by Cormack [1]. Cormack's method (CM), adopted for symmetry systems [12], has been applied many times for reconstructing electron-positron momentum densities from 2D ACAR data [13-28] as well as for reconstructing line dimensions of the Fermi surface from Compton scattering profiles (i.e. conversion: from $1 \mathrm{D}$ to 2D) [29-32].

For $N=3$ (plane integrals) the Gegenbauer transform reduces to the Legendre transform, and choosing $\lambda=N / 2$ Luis formulae become [33]:

$$
g_{l}(r)=\sum_{k=0}^{\infty} a_{l k}\left(1-p^{2}\right) P_{l+2 k}^{(1,1)}(r)
$$


and

$$
\rho_{l}(p)=\frac{1}{\pi} \sum_{k=0}^{\infty} a_{l k}(1+2 k+l) p^{l} P_{k}^{(0, l+1 / 2)}\left(2 p^{2}-1\right) .
$$

Such a technique [33] up to now has been applied to reconstructing electron densities from the Compton scattering spectra in yttrium [34], $\mathrm{Cu}_{0.1} \mathrm{Al}_{0.9}$ [35], and to the shape-memory alloy $\mathrm{Ni}_{0.62} \mathrm{Al}_{0.38}[36]$.

2.2. Solution in terms of Hermite $(H)$ and Laguerre $(L)$ polynomials

The second set of polynomials are

$$
g_{l}(r)=\sum_{n} a_{n l} \mathrm{e}^{-p^{2}} H_{l+2 n}(r)
$$

and

$$
\rho_{l}(p)=\frac{1}{\pi^{\mu+1 / 2}} \mathrm{e}^{-p^{2}} p^{l} \sum_{n}(-1)^{n} n ! 2^{2 n+l} a_{n l} L_{n}^{l+\mu}\left(p^{2}\right) .
$$

Such a set of polynomials for $N=2$ was firstly derived by Cormack [1] and for $N=3$ noticed by Mijnarends [2] who (due to poor computer abilities in 1967) proposed quite another solution by using a Fourier-Hankel transform to Eq. (4). Mijnarends' method has been applied many times to 1D ACAR data [37] and Compton scattering profiles [38].

The above solution (Eq. (9)) for $N=3(\mu=1 / 2)$ was next proposed by Reiter and Silver [39] (and also in Ref. [40]) and employed to neutron scattering experiments [41, 42].

\section{Fourier transform (FT) methods}

In the FT techniques [43] one calculates the 1D FT of a measured spectrum $g$ :

$$
f(q, \boldsymbol{\zeta})=2 \int_{0}^{\infty} g(r, \boldsymbol{\zeta}) \cos (2 \pi r q) \mathrm{d} r
$$

getting in this way either $2 \mathrm{D}$ or $3 \mathrm{D}$ FT of $\rho(\boldsymbol{p})$ for $N=2$ and $N=3$, respectively. Next, the reconstructed density is evaluated from the inverse FT. For line projections it could be written in the form

$$
\rho(p, \Theta)=\int_{0}^{\pi} W[p \cos (\Theta-\varphi), \varphi] \mathrm{d} \varphi
$$

where

$$
W(s, \varphi)=2 \int_{0}^{\infty} F(q, \varphi)|q| \cos (2 \pi s q) \mathrm{d} q .
$$

As shown by Brooks and Di Chiro [3], two integrals given by Eqs. (10) and (12) can be reduced to one integral and further replaced by the following summation:

$$
W\left(s_{i}, \varphi\right)=\frac{g\left(s_{i}, \varphi\right)}{4 \Delta s}-\frac{1}{\pi^{2} \Delta s} \sum_{j=1}^{n} \frac{g\left(s_{j}, \varphi\right)}{(i-j)^{2}},
$$

over all $j$ 's for which $i-j$ is odd, where $\Delta s$ denotes the distance between exper- 
imental points. This method, applied to first 2D ACAR measurements [44-49], is named filtered back projection (FBP) with a reference to the back projection operation (e.g. [43]) where

$$
\rho(p, \Theta)=\int_{0}^{\pi} g[p \cos (\Theta-\varphi), \varphi] \mathrm{d} \varphi .
$$

It was generally supposed that Fourier transform methods need a knowledge of many projections [44-49], whereas, as shown in the paper [12], the CM allows to reconstruct $\rho(\boldsymbol{p})$ basing on a small number of measured spectra. It was explained in the paper [50] where we showed that the necessity of integration with a small $\Delta \varphi$ (Eq. (11)) is not equivalent to the knowledge of a great number of projections. In positron annihilation experiments it is sufficient to measure a few projections and introduce the angular interpolation (Eqs. (2), (3)) either to experimental data or to the function $W(s, \varphi)$. A knowledge of radial components $W_{n}$ allows to estimate $W(s, \varphi)$ for arbitrary angle $\varphi$, so to perform the integration in Eq. (9) with an arbitrarily small $\Delta \varphi$. Such a method was applied (parallel with the CM) in the papers $[17,20-26]$. There is also another possibility. Introducing the angular interpolation into $F(q, \varphi)$ eliminates the integration over the angle $\varphi$ and integrals (10), (11) become (e.g. [43]):

$$
\rho_{n}(p)=2 \pi(\mathrm{i})^{n} \int_{0}^{1} F_{n}(q)|q| J_{n}(2 \pi p q) \mathrm{d} q,
$$

where $J_{n}$ denotes the Bessel function of the first kind of the order $n$ and

$$
F_{n}(q)=2 \int_{0}^{1} g_{n}(r) \cos (2 \pi r q) \mathrm{d} p,
$$

with $g_{n}$ and $\rho_{n}$ defined in Eqs. (2), (3). Such a procedure, called the FourierBessel (F-B) method, was applied to reconstruct 2D densities from 2D ACAR data [51] and 3D densities from 1D Compton profiles [52-56] (for 3D space $|q|$ in Eq. (11) should be replaced by $q^{2}$ ). Some modification of the F-B method (expansion of radial components in a series of Gaussian-based function) was proposed in Ref. [57]. However, because calculations of the Bessel functions of a higher order make some difficulties, lately the direct FT (instead of F-B) algorithm [58] has been used both to 2D ACAR [59-63] as well as to 1D Compton profiles reconstructing either fully 3D densities [64-69] or 2D ones [70-73]. Such techniques involving both fast FT algorithm as well as different ways of interpolations were elaborated by many authors, see e.g. [74].

When measured 2D spectra are not collected in such a way that the reconstruction of a $3 \mathrm{D}$ density from its $2 \mathrm{D}$ projections can be reduced to a set of reconstructions of $2 \mathrm{D}$ densities, performed independently on parallel planes, one can use e.g. the Pecora method [75]. In this technique, applied in papers [76-79], one calculates the 1D FT of measured 2D spectra which represent the 3D FT of the 3D density. By expanding FT into the lattice harmonics, one reconstructs the 3D density according to the Fourier-Bessel method proposed for plane pro- 
TABLE

Reconstruction techniques applied to ACAR and CP spectra.

\begin{tabular}{|c|c|c|}
\hline Experiment $\Rightarrow$ reconstruction & $\begin{array}{c}\text { Applied } \\
\text { technique }\end{array}$ & References \\
\hline 1D ACAR $\Rightarrow 3 \mathrm{D}$ densities & MM & {$[2]$ and 6 papers in $[37]$} \\
\hline \multirow[t]{6}{*}{$2 \mathrm{D} \mathrm{ACAR} \Rightarrow 3 \mathrm{D}$ densities } & FBP & {$[44-49]$} \\
\hline & $\mathrm{PM}$ & {$[76-79]$} \\
\hline & $\mathrm{CM}$ & {$[13-28]$} \\
\hline & $\mathrm{F}-\mathrm{B}$ & {$[51]$} \\
\hline & DFT & {$[58-62]$ and 14 in [63] } \\
\hline & MFBP & {$[17,20-26]$} \\
\hline \multirow[t]{5}{*}{ 1D $\mathrm{CP} \Rightarrow 3 \mathrm{D}$ densities } & MM & 2 papers in [38] \\
\hline & $\mathrm{F}-\mathrm{B}$ & {$[53-57]$} \\
\hline & JP & {$[34-36]$} \\
\hline & DFT & {$[64-69]$} \\
\hline & $\mathrm{ME}$ & {$[82-83]$} \\
\hline \multirow[t]{2}{*}{$1 \mathrm{D} \mathrm{CP} \Rightarrow 2 \mathrm{D}$ densities } & $\mathrm{CM}$ & {$[29-32]$} \\
\hline & DFT & {$[70-73]$} \\
\hline $1 \mathrm{D} N \mathrm{NS} \Rightarrow 3 \mathrm{D}$ densities & $\mathrm{HP}$ & {$[41,42]$} \\
\hline
\end{tabular}

jections $[52,53]$. This method is equivalent to the following procedure. For each of $2 \mathrm{D}$ spectrum one can estimate some number of $1 \mathrm{D}$ spectra $g\left(p_{z}\right)$ for different directions $p_{z}$ applying either the Fourier-Bessel method or another algorithm for reconstructing densities from plane projections. We apply such a procedure with using the Jacobi polynomials technique (Eq. (8)) in the papers [33, 34]. Having twelve 2D ACAR spectra for Gd [46], we reconstructed $\rho(\boldsymbol{p})$ applying Cormack's method [1]. Next, we created 1D ACAR profiles, performing the reconstruction using the Jacobi polynomial technique. In this way we made a test of the proposed technique as well we studied the question which projections should be measured in the Compton experiment in order to reconstruct densities most efficiently [33]. The same procedures were applied to 2D ACAR data in yttrium before performing the high resolution Compton scattering experiment in this metal in order to decide which profiles should be measured [34].

In the case of line projections the best solution is to perform the reconstruction of $2 \mathrm{D}$ densities $\rho(\boldsymbol{p})$ independently of parallel planes. Having them, in the case of cubic structures, one can either make a symmetrization or use a filtering procedure as applied to $\mathrm{LaB}_{6}[24]$ (detailed description is given in [80]).

\section{Summary}

We found about 90 papers where different reconstruction techniques were applied to study electron momentum densities from 1D ACAR, 2D ACAR, 1D 
Compton (or neutron) scattering experimental spectra. These techniques belong to transform methods except of the maximum entropy algorithm [81], applied to 1D Compton profiles [82, 83]. This algorithm, which belongs to series expansion methods (see [9] and references therein) and is known as an algebraic technique, was proposed for image reconstruction starting from 1979 [84].

This is summarized in Table, where the following abbreviations are used: CM - Cormack method (with orthogonal Chebyshev polynomials) [1, 12]; DFT - direct Fourier transform [58, 74]; FBP - filtered back projection [3] using Eq. (13); F-B — Fourier Bessel [52, 53]; HP — Hermite polynomials [39]; JP Jacobi polynomials [33]; ME - maximum entropy [81, 84]; MFBP — modified FBP [50]; MM — Mijnarends' method [2]; PM — Pecora method [75].

This paper makes a first step towards an overview paper where we will give information about investigated materials and main results of papers quoted in Table. We will also discuss an optimal strategy of corresponding measurements, i.e. 1 - how many projections and which ones should be measured; 2 - which reconstruction technique is the most efficient for such kind of studies as well as 3 - distribution of the experimental noise and 4 - a way of data improvement (consistency conditions, deconvoluting and filtering procedures). Some of these questions are discussed in Refs. $[52,85]$.

\section{Acknowledgments}

We are grateful to Dr. M. Wolf for helpful discussions.

\section{References}

[1] A.M. Cormack, J. Appl. Phys. 34, 2722 (1963); 35, 2908 (1964).

[2] P.E. Mijnarends, Phys. Rev. 160, 512 (1967).

[3] A. Brooks, G. Di Chiro, Phys. Med. Biol. 21, 689 (1976).

[4] G.T. Herman, Image Reconstruction from Projection, Academic, New York 1980.

[5] S.R. Deans, The Radon Transform and Some of Its Applications, Wiley, New York 1983.

[6] F. Natterer, The Mathematics of Computerized Tomography, Wiley, Stuttgart 1986.

[7] J. Radon, Berichte Sachsische Akademie der Wissenschaften 69, 262 (1917).

[8] F. John, Plane Waves and Spherical Means Applied to Partial Differential Equations, Interscience, New York 1955.

[9] Y. Censor, Proc. IEEE 71, 409 (1983).

[10] R.M. Lewitt, Proc. IEEE 71, 390 (1983).

[11] A.K. Luis, SIAM J. Math. Anal. 15, 621 (1984).

[12] G. Kontrym-Sznajd, Phys. Status Solidi A 117, 227 (1990).

[13] G. Kontrym-Sznajd, J. Majsnerowski, Solid State Commun. 70, 593 (1989).

[14] G. Kontrym-Sznajd, J. Majsnerowski, J. Phys. CM 2, 9927 (1990). 
[15] S.B. Dugdale, H.M. Fretwell, M.A. Alam, G. Kontrym-Sznajd, R.N. West, S. Badrzadeh, Phys. Rev. Lett. 79, 941 (1997).

[16] H.M. Fretwell, S.B. Dugdale, M.A. Alam, D.C.R. Hedley, A. Rodriguez-Gonza'alez, S.B. Palmer, Phys. Rev. Lett. 82, 3867 (1999).

[17] M. Biasini, G. Kontrym-Sznajd, M.A. Monge, M. Gemmi, A. Czopnik, A. Jura, Phys. Rev. Lett. 86, 4616 (2001)

[18] I. Wilkinson, R.J. Hughes, Zs. Major, S.B. Dugdale, M.A. Alam, E. Bruno, B. Ginatempo, E.S. Giuliano, Phys. Rev. Lett. 87, 216401 (2001).

[19] A. Jura, G. Kontrym-Sznajd, M. Samsel-Czekała, J. Phys. Chem. Solids 62, 2241 (2001).

[20] M. Biasini, G. Ferro, G. Kontrym-Sznajd, A. Czopnik, Phys. Rev. B 66, 075126 (2002).

[21] M.A. Monge, M. Biasini, G. Ferro, M. Gemmi, G. Satta, S. Massidda, P. Lejay, A. Continenza, Phys. Rev. B 65, 035114 (2002).

[22] M. Biasini, G. Ferro, A. Czopnik, Phys. Rev. B 68, 094513 (2003).

[23] J. Rusz, M. Biasini, A. Czopnik, Phys. Rev. Lett. 93, 156405 (2004).

[24] M. Biasini, M.A. Monge, G. Kontrym-Sznajd, H. Harima, N. Sato, Mater. Sci. Forum 363-365, 582 (2001); G. Kontrym-Sznajd, M. Samsel-Czekała, M. Biasini, Y. Kubo, Phys. Rev. B 70, 125103 (2004).

[25] S.J. Crowe, S.B. Dugdale, Zs. Major, M.A. Alam, J.A. Duffy, S.B. Palmer, Europhys. Lett. 65, 285 (2004).

[26] G. Kontrym-Sznajd, M. Samsel-Czekała, M. Biasini, Acta Phys. Pol. A 113, 1429 (2008).

[27] Zs. Major, S.B. Dugdale, R.J. Watts, G. Santi, M.A. Alam, S.M. Hayden, J.A. Duffy, J.W. Taylor, T. Jarlborg, E. Bruno, D. Benea, H. Ebert, Phys. Rev. Lett. 92, 107003 (2004).

[28] R.J. Hughes, S.B. Dugdale, Zs. Major, M.A. Alam, T. Jarlborg, E. Bruno, B. Ginatempo, Phys. Rev. B 69, 174406 (2004).

[29] G. Kontrym-Sznajd, R.N. West, S.B. Dugdale, Mater. Sci. Forum 255-257, 796 (1997).

[30] S.B. Dugdale, H.M. Fretwell, K.J. Chen, Y. Tanaka, A. Shukla, T. Buslaps, Ch. Bellin, G. Loupias, M.A. Alam, A.A. Manuel, P. Suortti, N. Shiotani, J. Phys. Chem. Solids 61, 361 (2000).

[31] G. Kontrym-Sznajd, M. Samsel-Czekała, S. Huotari, K. Hämäläinen, S. Manninen, Phys. Rev. B 68, 155106 (2003).

[32] G. Kontrym-Sznajd, M. Samsel-Czekała, Appl. Phys. A 80, 1693 (2005).

[33] G. Kontrym-Sznajd, M. Samsel-Czekała, Appl. Phys. A 70, 89 (2000).

[34] G. Kontrym-Sznajd, M. Samsel-Czekała, A. Pietraszko, H. Sormann, S. Manninen, S. Huotari, K. Hämäläinen, J. Laukkanen, R.N. West, W. Schülke, Phys. Rev. B 66, 155110 (2002).

[35] M. Samsel-Czekała, G. Kontrym-Sznajd, G. Döring, W. Schülke, J. Kwiatkowska, F. Maniawski, S. Kaprzyk, A. Bansil, Appl. Phys. A 76, 87 (2003). 
[36] S.B. Dugdale, R.J. Watts, J. Laverock, Zs. Major, M.A. Alam, M. Samsel-Czekała, G. Kontrym-Sznajd, Y. Sakurai, M. Itou, D. Fort, Phys. Rev. Lett. 96, 046406 (2006).

[37] P.E. Mijnarends, Phys. Rev. 178, 622 (1969); H. Stachowiak, Phys. Status Solidi 41, 599 (1970); G. Kontrym-Sznajd, H. Stachowiak, Appl. Phys. 5, 361 (1975); G. Kontrym-Sznajd, H. Stachowiak, W. Wierzchowski, K. Petersen, N. Thrane, G. Trumpy, Appl. Phys. 8, 151 (1975); St. Chabik, J. Pająk, B. Rozenfeld, G. Kontrym-Sznajd, Acta Phys. Pol. A 50, 623 (1976); G. Kontrym-Sznajd, M. Šob, H. Stachowiak, Czech. J. Phys. B 29, 1124 (1979).

[38] P.E. Mijnarends, Phys. Rev. B 4, 2820 (1971); W. Schülke, Phys. Status Solidi B 62, 453 (1974).

[39] G. Reiter, R. Silver, Phys. Rev. Lett. 54, 1047 (1985).

[40] G.P. Das, K.V. Bhagwat, V.C. Sahni, Phys. Rev. A 36, 2984 (1987).

[41] G.F. Reiter, J. Mayers, P. Platzman, Phys. Rev. Lett. 89, 135505 (2002).

[42] G.F. Reiter, J. Mayers, J. Noreland, Phys. Rev. B 65, 104305 (2002).

[43] R.M. Lewitt, R.H. Bates, Optik 50, 19 (1978); Optik 50, 85 (1978); Optik 50, 189 (1978).

[44] F. Sinclair, W.S. Farmer, S. Berko, in: Positron Annihilation, Eds. P.G. Coleman, S. Sharma, L.M. Diana, North-Holland Publ. Co, Amsterdam 1982, p. 322.

[45] K.R. Hoffmann, S. Berko, in Ref. [44], p. 325.

[46] R.L. Waspe, R.N. West, in Ref. [44], p. 328.

[47] A.A. Manuel, L. Oberli, T. Jarlborg, R. Sachot, P. Descouts, M. Peter, in Ref. [44], p. 281.

[48] M.A. Alam, R.L. Waspe, R.N. West, in: Positron Annihilation, Eds. L. Dorikens-Vanpraet, M. Dorikens, D. Segers, World Sci., Singapore 1989, p. 242.

[49] T. Jarlborg, A.A. Manuel, M. Peter, Phys. Rev. B 27, 4210 (1983).

[50] G. Kontrym-Sznajd, E. Józefczuk, Mater. Sci. Forum 255-257, 754 (1997).

[51] T. Chiba, Y. Nagai, Z. Tang, T. Akahane, M. Hasegawa, M. Takenaka, E. Kuramoto, F-B method, paper presented during ICPA-14, Ontario, Canada 2007.

[52] P.E. Mijnarends, in: Compton Scattering, Ed. B. Williams, McGraw-Hill, London 1977, Ch. 10, p. 323.

[53] N.K. Hansen, HMI-Report, B342 (1980); N.K. Hansen, P. Pattison, J.R. Schneider, Z. Phys. B 66, 305 (1987).

[54] W. Schülke, F. Wohlert, A. Kaprolat, Phys. Rev. B 54, 14381 (1996).

[55] G. Stutz, F. Wohlert, A. Kaprolat, W. Schülke, M. Ito, H. Kawata, N. Shiotani, S. Kaprzyk, A. Bansil, Phys. Rev. B 60, 7099 (1999).

[56] D.N. Timms, M.J. Cooper, R.S. Holt, F. Itoh, T. Kobayashi, H. Nara, J. Phys. CM 2, 10517 (1990).

[57] J.M. Gillet, C. Fluteaux, P.J. Becker, Phys. Rev. B 60, 2345 (1999).

[58] R. Suzuki, S. Tanigawa, in: Positron Annihilation, Eds. P.C. Jain, R.M. Singru, K.P. Gopinathan, World Sci., Singapore 1985, p. 626. 
[59] S. Tanigawa, T. Kurihara, M. Osawa, T. Komatsubara, Y. Onuki, in Ref. [58], p. 239; S. Tanigawa, Y. Mizuhara, T. Kurihara, M. Oda, Y. Enomoto, M. Suzuki, T. Murakami, in Ref. [58], p. 933.

[60] R. Suzuki, S. Tanigawa, N. Shiotani, M. Matsumoto, S. Wakoh, in Ref. [58], p. 245; R. Suzuki, M. Osawa, S. Tanigawa, M. Matsumoto, N. Shiotani, J. Phys. Soc. Japan 58, 3251 (1989).

[61] H. Nakashima, T. Kubota, H. Kondo, Y. Murakami, S. Tanigawa, Phys. Status Solidi B 170, 171 (1992).

[62] H. Kondo, T. Kubota, H. Nakashima, Y. Murakami, S. Tanigawa, Phys. Status Solidi B 177, 345 (1993).

[63] 14 papers published by Tanigawa group, in: Positron Annihilation, Eds. Zs. Kajcos, Cs. Szeles, Trans. Tech. Publ., Switzerland 1992, pp 615, 619, 675, 679, 683, 687, 719, 723, 727, 771, 775, 779, 783, 1997.

[64] Y. Tanaka, N. Sakai, Y. Kubo, H. Kawata, Phys. Rev. Lett. 70, 1537 (1993).

[65] Y. Tanaka, Y. Sakurai, A.T. Stewart, N. Shiotani, P.E. Mijnarends, S. Kaprzyk, A. Bansil, Phys. Rev. B 63, 045120 (2001).

[66] N. Shiotani, I. Matsumoto, H. Kawata, J. Katsuyama, M. Mizuno, H. Araki, Y. Shirai, J. Phys. Soc. Japan 73, 1627 (2004).

[67] I. Matsumoto, J. Kwiatkowska, F. Maniawski, M. Itou, H. Kawata, N. Shiotani, S. Kaprzyk, P.E. Mijnarends, B. Barbiellini, A. Bansil, Phys. Rev. B 64, 045121 (2001).

[68] I. Matsumoto, H. Kawata, N. Shiotani, Phys. Rev. B 64, 195132 (2001).

[69] N. Hiraoka, T. Buslaps, V. Honkimäki, H. Minami, H. Uwe, Phys. Rev. B 75, 121101 (2007).

[70] A. Koizumi, T. Nagao, N. Sakai, K. Hirota, Y. Murakami, Phys. Rev. B 74, 012408 (2006).

[71] N. Hiraoka, A. Deb, M. Itou, Y. Sakurai, Z.Q. Mao, Y. Maeno, Phys. Rev. B 67, 094511 (2003); N. Hiraoka, T. Buslaps, V. Honkimäki, H. Minami, H. Uwe, Phys. Rev. B 71, 205106 (2005).

[72] N. Hiraoka, T. Buslaps, V. Honkimäki, H. Guyot, C. Schlenker, Phys. Rev. B 71, 125417 (2005); N. Hiraoka, T. Buslaps, V. Honkimki, T. Nomura, M. Itou, Y. Sakurai, Z.Q. Mao, Y. Maeno, Phys. Rev. B 74, 100501(R) (2006).

[73] Y. Tanaka, K.J. Chen, Ch. Bellin, G. Loupias, H.M. Fretwell, A. Rodrigues-Gonzalez, M.A. Alam, S.B. Dugdale, A.A. Manuel, A. Shukla, T. Buslaps, P. Suortti, N. Shiotani, J. Phys. Chem. Solids 61, 365 (2000).

[74] R.M. Mersereau, Comput. Biol. Med. 6, 247 (1976); H. Choi, D.C. Munson Jr., Int. J. Imaging Systems Technol. 9, 1 (1998).

[75] L.M. Pecora, IEEE Trans. Nucl. Sci. NS-34, 642 (1987); J. Phys. CM 1, SA1 (1989).

[76] M. Pecora, A.C. Ehrlich, A.A. Manuel, A.K. Singh, R.M. Singru, in Ref. [58], p. 254.

[77] L.M. Pecora, A.C. Ehrlich, A.A. Manuel, A.K. Singh, M. Peter, R.M. Singru, Phys. Rev. B 37, 6772 (1988). 
[78] H.M. Fretwell, S.B. Dugdale, M.A. Alam, M. Biasini, L. Hoffmann, A.A. Manuel, Europhys. Lett. 32, 771 (1995).

[79] W. Triftshäuser, E. Eckert, G. Kögel, P. Sperr, Mater. Sci. Forum 105-110, 501 (1992); S.S. Rajput, R. Prasad, R.M. Singru, W. Triftshäuser, A. Eckert, G. Kögel, S. Kaprzyk, A. Bansil, J. Phys. CM 5, 6419 (1993).

[80] G. Kontrym-Sznajd, M. Samsel-Czekała, Appl. Phys. A 89, 975 (2007).

[81] L. Dobrzyński, A. Holas, Nucl. Instrum. Methods Phys. Res., Sect. A 383, 589 (1996).

[82] L. Dobrzyński, E. Żukowski, J. Phys. CM 11, 8049 (1999).

[83] Ch. Bellin, L. Dobrzyński, H. Kouba, G. Loupias, T. Buslaps, V. Honkimki, Z. Phys. Chem. 215, 1367 (2001).

[84] G. Minerbo, Comp. Graph. Imag. Proc. 10, 48 (1979); Comput. Biol. Med. 9, 29 (1979).

[85] X-ray Compton Scattering, Eds. M.J. Cooper, P.E. Mijnarends, N. Shiotani, N. Sakai, A. Bansil, Oxford University Press, Oxford 2004. 